\title{
The broad-band X-ray spectrum of the blazar PKS B1830-211 by Chandra and INTEGRAL
}

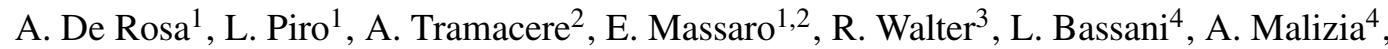 \\ A. J. Bird ${ }^{5}$, and A. J. Dean ${ }^{5}$ \\ 1 IASF - Sezione di Roma, INAF, via del Fosso del Cavaliere 100, 00113 Roma, Italy \\ e-mail: derosa@rm.iasf.cnr.it \\ 2 Dipartimento di Fisica, Università La Sapienza, Piazzale A. Moro 2, 00185 Roma, Italy \\ 3 Geneva Observatory, INTEGRAL Science Data Centre, Chemin d'Ecogia 16, 1291 Versoix, Switzerland \\ 4 IASF - Sezione di Bologna, INAF, via P. Gobetti 101, 40129 Bologna, Italy \\ 5 School of Physics and Astronomy, University of Southampton, Highfield, Southampton, SO17 1BJ, UK
}

Received 17 January 2005 / Accepted 5 April 2005

\begin{abstract}
In this paper we present a broad-band study of the X-ray emission of the blazar PKS 1830-211 based on Chandra and INTEGRAL observations. Notwithstanding the high redshift $(z=2.507)$, it is a bright $\mathrm{X}$-ray source $\left(F(2-10 \mathrm{keV}) \simeq 10^{-11} \mathrm{erg} \mathrm{cm}^{-2} \mathrm{~s}^{-1}\right)$, due to gravitational lensing by an intervening galaxy at $z=0.89$. Previous X-ray observations attribute the observed absorption at $E<2 \mathrm{keV}$ to the lensing galaxy. Our analysis, although not in contrast with this hypothesis, suggests also the possibility of an intrinsic (ionized) absorption, taking place at the front side of the jet. This scenario is also supported by some evidence, in the same data, of a feature observed at $2.15 \mathrm{keV}$ which can be interpreted as a blueshifted iron line $(v / c \simeq 0.18)$. The SED of PKS 1830-211 can be well modelled by combining a Synchrotron Self-Compton component and an external source of photons to be scattered up to $\gamma$-ray energies by relativistic electrons moving outward in the jet. The main source of low energy photons is a dust torus at the temperature of $10^{3} \mathrm{~K}$ as expected in $\mathrm{MeV}$ blazars.
\end{abstract}

Key words. radiation mechanisms: non-thermal - galaxies: active - X-rays: galaxies: individual: PKS B1830-211

\section{Introduction}

PKS B1830-211 is a high redshift blazar $(z=2.507$, Lidman et al. 1999) gravitationally lensed by an intervening galaxy at $z=0.89$. Its radio image shows two compact components separated by about $1^{\prime \prime}$ and believed to arise from the core of the source (hereafter North-East NE and South-West SW components), plus a ringlike extended structure arising from the jet (Wiklind \& Combes 1996), connecting the compact components (Nair et al. 1993; Pramesh Rao \& Subrahmanyan 1988). In the radio band the source shows strong variability (Lovell et al. 1998) and has a flat spectrum. It was also observed in the infrared (Lidman et al. 1999), in X-rays (Mathur \& Nair 1997; Oshima et al. 2001) and in $\gamma$-rays (Mattox et al. 1997; Bassani et al. 2004).

ROSAT data showed that the X-ray spectrum is best represented by a power law absorbed at the redshift of the lens galaxy by a column density $N_{\mathrm{H}}=(3.5 \pm 0.5) \times 10^{22} \mathrm{~cm}^{-2}$; no additional absorption component was required, suggesting that both lensed images are covered by the same absorber (Mathur \& Nair 1997). On the contrary, ASCA data showed that the spectrum is consistent with two absorption components having different column densities: $N_{\mathrm{H}}^{\text {low }}<1.5 \times 10^{22} \mathrm{~cm}^{-2}$ and $N_{\mathrm{H}}^{\text {high }}=$ $\left(7.5 \pm_{-0.9}^{+0.8}\right) \times 10^{22} \mathrm{~cm}^{-2}$ (Oshima et al. 2001), interpreted by the authors considering that the low- and high-absorbing component correspond to the NE and SW lensed images, respectively. The ratio of the two magnification factors was $0.21_{-0.08}^{+0.07}$, smaller than the value found at radio wavelengths which was $1.55 \pm 0.02$ (Lovell et al. 1998). In this paper we investigate the broad-band X-ray spectrum of PKS 1830-211 observed by Chandra and INTEGRAL. In Sect. 2 we describe the observations, while data analysis is presented in Sect. 3. In Sect. 4 the nature of the complex absorber is discussed, and in Sect. 5 the SED of PKS 1830-211 is derived and a possible emission model is presented. We summarize our results and conclusions in Sect. 6.

\section{Observations and data reduction}

\subsection{INTEGRAL}

PKS1830-211 is one of the AGNs detected by INTEGRAL in the Galactic Center region (Bassani et al. 2004). It has been reported as an ISGRI source by Bird et al. (2004) and Revinivtsev et al. (2004) and at a redshift of 2.507 it is the furthest object so far detected by INTEGRAL. The observational data reported here refer to the first $I B I S$ survey which consists of several pointings carried out between 2003 February 28 to October 10. 


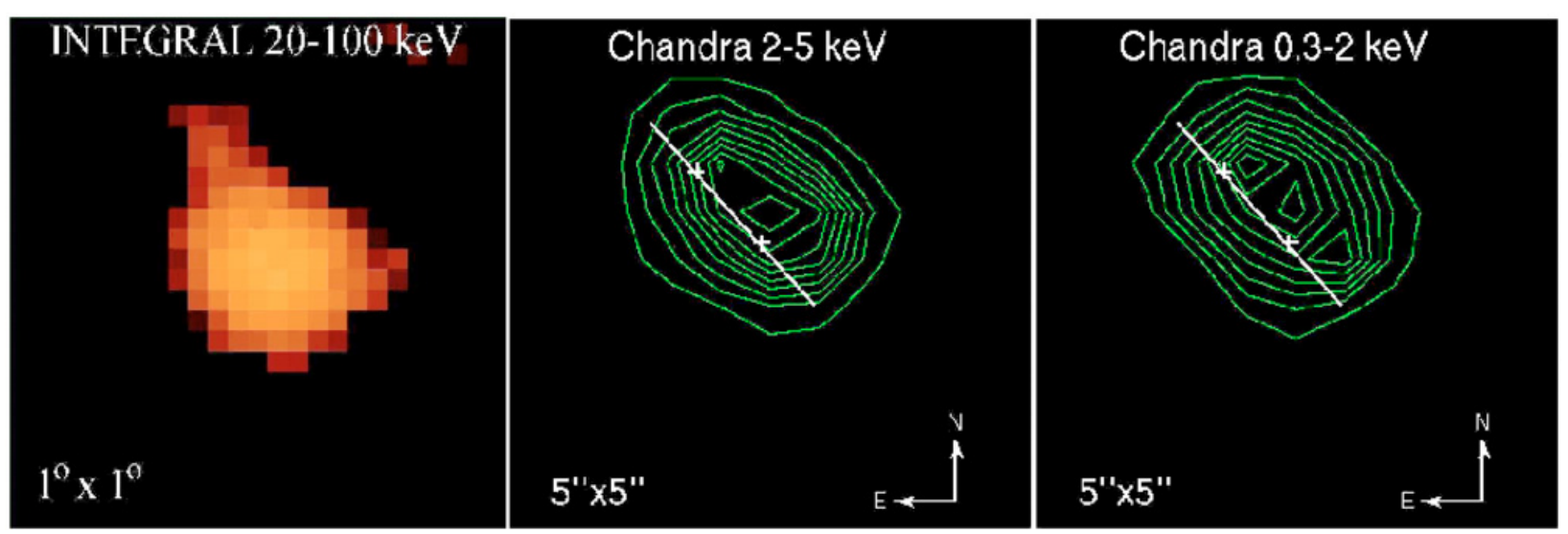

Fig. 1. X-ray images of PKS 1830-211. The INTEGRAL image is shown in the left panel, its extension is compatible with the PSF of ISGRI. In the middle and right panel we show the Chandra contour levels in two different energy range together with the position of the NE and SW lobes from radio observations (Pramesh Rao \& Subrahmanyan 1988). The two main components of the lensing are distinguished in Chandra images.

The total on source exposure is $714 \mathrm{ks}$, providing a detection at $\sim 14 \sigma$ confidence level. The mean counting rate is $0.29 \pm 0.03$ counts $\mathrm{s}^{-1}$ both in the $20-40$ and $40-100 \mathrm{keV}$ band, a clear indication of a hard spectrum. These count rates correspond to a flux of roughly 3 (2.4) and 4 (3.9) $\mathrm{mCrab}\left(10^{-11} \mathrm{erg} \mathrm{cm}^{-2} \mathrm{~s}^{-1}\right)$ in the two bands, respectively. ISGRI images for each available pointing were generated in narrow energy bands using the ISDC offline scientific analysis software OSA version 3.0 (Goldwurm et al. 2003), including background uniformity corrections (Terrier et al. 2003). Source ghosts have been removed from each image using a catalogue built iteratively and finally containing all sources detected. The individual images were then combined to produce a mosaic of the region of interest in broader bands to enhance the detection significance using the system described in detail by Bird et al. (2004).

The combined 20-100 keV image of the source is shown in the left panel in Fig. 1. The ISGRI image is point-like, and the extension is compatible with the PSF of the instrument.

\subsection{Chandra}

Chandra observations of PKS B1830-211 were performed on two occasions one year apart, in 2000 June 26-27 and 2001 June 25 , for a total exposure of about $50 \mathrm{ks}$. The source did not show evidence of variability between the two pointings. The good spatial resolution allows us to observe the two lensed images, with an angular distance of the order of the Chandra resolution limit $\left(\sim 1^{\prime \prime}\right)$. In Fig. 1 we show the ACIS contour levels extracted in two separate energy ranges $0.3-2 \mathrm{keV}$ (right panel) and $2-5 \mathrm{keV}$ (middle panel). In the figure we indicate also the position of the NE and SW components taken from radio observation (Pramesh Rao \& Subrahmanyan 1988). The offset between radio and X-rays image is less than $1^{\prime \prime}$. There is evidence for an elliptical shape in both images, as showed by the plotted contours, and the two main components of the lensing effect are visible. In Fig. 2 we plot the X-ray intensity profiles along the major axis of the ellipse which shows that it is significantly larger than the ACIS PSF. The ACIS PSF is very sharply

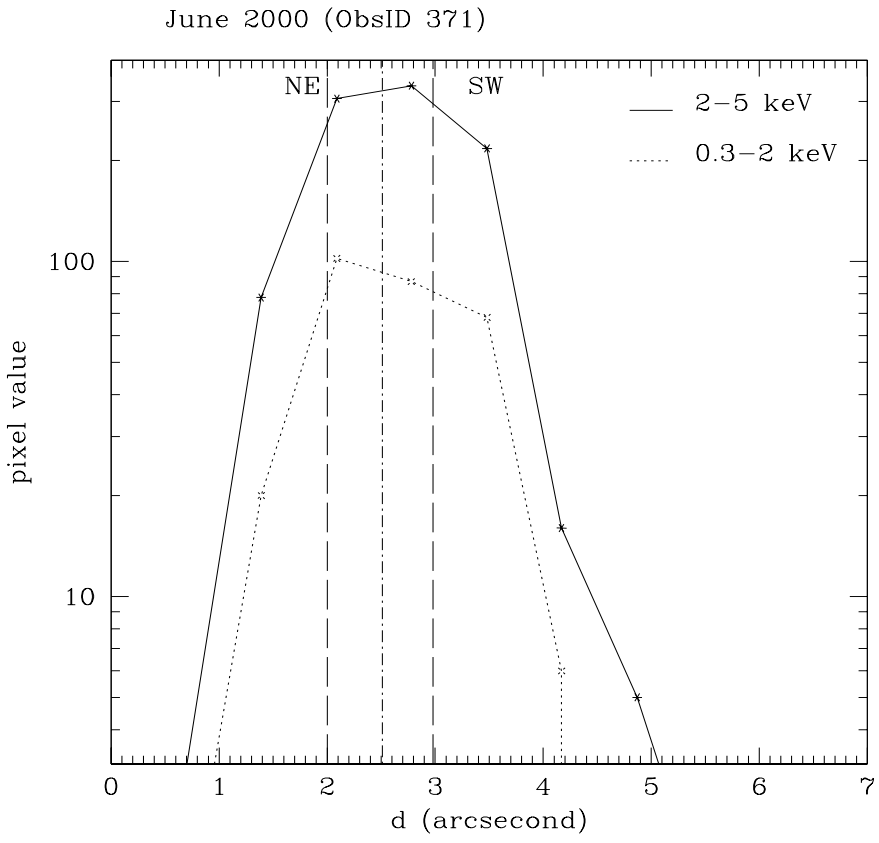

Fig. 2. X-ray intensity profiles along the major axis of the ellipse for the Chandra observation in 2000 June.

peaked on-axis, with a FWHM less than half an $\operatorname{arcsec}^{1}$. The ratio between the counts from the $\mathrm{NE}$ and $\mathrm{SW}$ components is about 1.5 in both $0.3-2 \mathrm{keV}$ and $2-5 \mathrm{keV}$ energy ranges, similar to the value observed at radio frequencies. This result indicates that the absorbing gas column densities of the two components cannot be very different, contrary to the findings of Courbin et al. (2002) in the optical and near-IR, where the SW component seems to be much weaker than the NE one.

\section{Spectral analysis and results}

Spectral analysis has been performed by fitting the data of both Chandra observations combined together, in fact fitting

\footnotetext{
${ }^{1}$ See The Chandra Proposers Observatory Guide (http://asc.harvard.edu/proposer/POG/html/MPOG.html)
} 


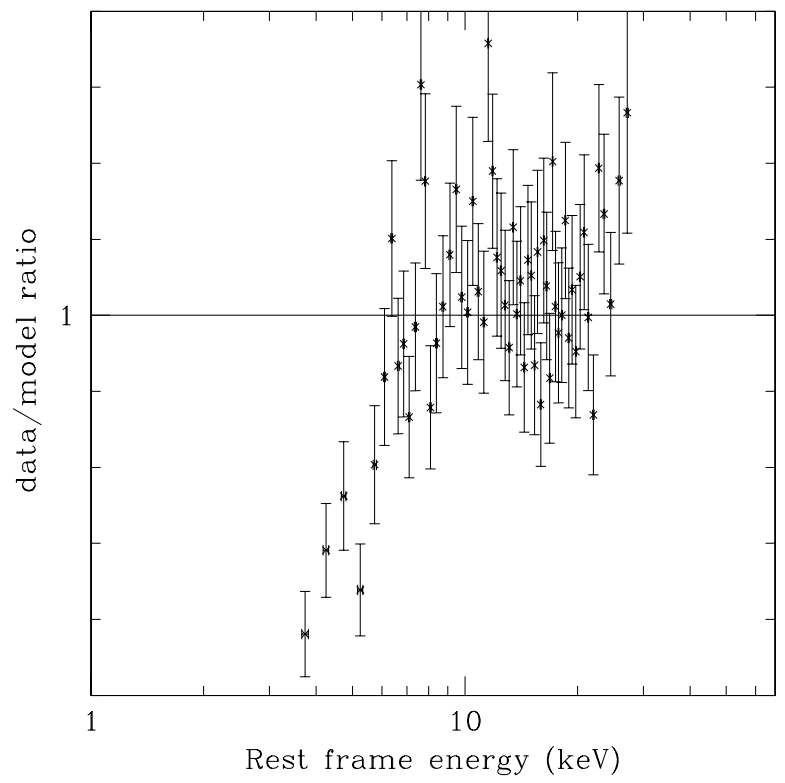

Fig. 3. Ratio of Chandra data (both observations combined) to a $\Gamma=1.02$ simple power-law (including Galactic absorption $N_{\mathrm{H}}^{\mathrm{Gal}}=$ $2.6 \times 10^{21} \mathrm{~cm}^{-2}$ and fitted over the $2-8 \mathrm{keV}$ range) in the source frame. Note the large residuals at energies below $5 \mathrm{keV}$.

them separately does not provide evidence for a significant difference. We first apply a simple power-law absorbed by Galactic gas $\left(N_{\mathrm{H}}^{\mathrm{Gal}}=2.6 \times 10^{21} \mathrm{~cm}^{-2}\right.$, Stark et al. 1992) in the whole range $0.5-8 \mathrm{keV}$ and obtain a poor fit with $\chi^{2} /$ d.o.f. $=725 / 459$. A better result is found fitting the data at energies higher than $2 \mathrm{keV}$, where the absorption is not relevant. In this case the above model gives a $\chi^{2} /$ d.o.f. $=389 / 377$ with a photon index $\Gamma=1.02 \pm 0.05$ and an estimated flux $F_{2-10} \simeq 10^{-11} \mathrm{erg} \mathrm{cm}^{-2} \mathrm{~s}^{-1}$. However, the data-to-model ratio in the energy range $2-8 \mathrm{keV}$ shows clear systematic residuals below $5 \mathrm{keV}$ in the source frame (Fig. 3). The INTEGRAL spectrum in the hard X-ray energy range $20-80 \mathrm{keV}$ is also well reproduced by this flat power-law model, confirming that the broad-band spectrum remains remarkably flat up to $80 \mathrm{keV}$. This will be discussed in more detail in the next section.

\section{The nature of the absorption}

Spectral flattening at energies less than $2 \mathrm{keV}$ has been found in radio-loud quasars having a redshift up to 4.4, using ASCA and XMM-Newton data (Fiore et al. 1998; Reeves \& Turner 2000; Worsley et al. 2004; Yuan et al. 2003, and references therein). Absorption has been suggested as the likely explanation for this flattening but the data collected up to now do not allow one to distinguish between an intrinsic absorber at the source redshift or an absorbing system along the line of sight. However, the UV-optical extinction observed in most of these objects is inconsistent with the high column density required to produce the flattening observed in X-rays. It was suggested that this gas could be ionized (Fabian et al. 2001), i.e. a warm absorber similar to that present in Seyfert 1 galaxies. Another possibility is that of an intrinsic low energy cut-off in the electron spectrum as proposed for other similar objects (Celotti \& Fabian 1993; Sikora et al. 1997).

First, we attempted to reproduce the low-energy spectral break with a cold absorber (in addition to the Galactic one) at the redshift of the lens galaxy $(z=0.89)$ as proposed by previous ROSAT (Mathur \& Nair 1997) and ASCA (Oshima et al. 2001) observations. The best fit values are reported in the first line of Table 1. Chandra data of both observations are well fitted with a single, flat power-law with photon index $\Gamma=1.09 \pm 0.05$, absorbed by a cold gas with $N_{\mathrm{H}} \simeq$ $2 \times 10^{22} \mathrm{~cm}^{-2}$ at $z=0.89\left(\chi^{2} /\right.$ d.o.f. $\left.=482 / 458\right)$. Data and the folded model are shown in Fig. 4 (left panel). INTEGRAL data, taken one year after Chandra observation, are also well reproduced by this continuum. The constant of cross-calibration between Chandra and INTEGRAL was free to vary during the fit and was found to be $C=0.57 \pm 0.13$. The broad-band spectrum of PKS 1830-211 is shown in Fig. 5, and the best fit values are reported in the last line of Table 1.

We also tested the possibility that the absorbing gas is intrinsic to the source, by fitting Chandra data with a warm absorber model. Best fit values are reported in the second line of Table 1 . The fit is good $\left(\chi^{2} /\right.$ d.o.f. $\left.=517 / 457\right)$ even if no better than that with a cold gas at $z=0.89$. This result suggests the presence of a mildly ionized gas with the column density $N_{\mathrm{H}} \simeq 10^{23} \mathrm{~cm}^{-2}$, and an upper limit to the ionization parameter $\xi=L_{2-10} / n R^{2}<100 \mathrm{erg} \mathrm{cm} \mathrm{s}^{-1}$, where $L_{2-10}$ is the inferred isotropic luminosity in the $2-10 \mathrm{keV}$ rest frame of the blazar while $n$ and $R$ are the absorber density and the distance from the source, respectively.

Chandra data show some evidence $(\approx 3 \sigma)$ in each observation of an emission feature around $2.15 \mathrm{keV}$ with an intensity of $2 \times 10^{-5} \mathrm{ph} \mathrm{cm}^{-2} \mathrm{~s}^{-1}$ and an equivalent width of about $50 \mathrm{eV}$ (see right panel in Fig. 4). The line is not resolved in the ACIS-S, setting the intrinsic width to be $<50 \mathrm{eV}$. ChandraHETG spectra are consistent with ACIS data, and set a lower limit on the intrinsic width to be $10 \mathrm{eV}$. It is important to note that the residuals around the line are of the order of a few tens of per cent, while the instrumental systematic effects at this energy are of the order of a few per cent. The energy of the line at the redshift of PKS 1830-211 is $7.54 \mathrm{keV}$, thus suggesting an association with a blueshifted iron line. This feature could be produced in the ambient matter around the source. If the line is due to neutral iron, the implied velocity for the gas has to be $v \simeq 0.18 c$. It is interesting to note that if the warm gas with a column density of $\sim 10^{23} \mathrm{~cm}^{-2}$ covers a solid angle of $2 \pi$ to the source, it should produce a line with $E W \simeq 50 \mathrm{eV}$, consistent with that detected in the Chandra spectrum.

\section{The spectral energy distribution}

We then used our spectral results and other data available in the literature to derive the SED of PKS 1830-211 shown in Fig. 6. Of course, it is not based on simultaneous observations and therefore must be considered an indicative picture of the emission properties of this source. $\gamma$-ray data are from the EGRET public archive (ftp://cossc.gsfc. nasa.gov/compton/data/egret/) and indicate a quite steep spectrum with a photon index of $2.58 \pm 0.13$. IR and 
Table 1. Results of the spectral fits of Chandra and INTEGRAL observations. Confidence ranges are 90 per cent for one parameter.

\begin{tabular}{lcclclcc}
\hline \hline & $\Gamma$ & ${ }^{1} N_{\mathrm{H}, z} /{ }^{1} N_{\mathrm{H}, \text { ion }}$ & ${ }^{2} \xi$ & ${ }^{3} F_{2-8 \mathrm{keV}}^{\text {una }}$ & ${ }^{3} F_{2-80 \mathrm{keV}}^{\text {una }}$ & ${ }^{3} F_{0.5-8 \mathrm{keV}}^{\text {una }}$ & $\chi^{2} /$ d.o.f. \\
\hline $0.5-8 \mathrm{keV}$ & $1.09_{-0.05}^{+0.05}$ & $1.93_{-0.26}^{+0.27}$ & - & 0.78 & 8.6 & 1 & $482 / 458$ \\
$0.5-8 \mathrm{keV}$ & $1.06_{-0.05}^{+0.05}$ & $9.5_{-2.1}^{+4.4}$ & $<100$ & 0.78 & 9.1 & 1 & $517 / 457$ \\
$0.5-80 \mathrm{keV}$ & $1.09_{-0.05}^{+0.05}$ & $1.94_{-0.25}^{+0.28}$ & - & 0.78 & 8.5 & 1 & $485 / 462$ \\
\hline
\end{tabular}

Note: ${ }^{(1)} N_{\mathrm{H}, z}$ is the column density at redshift of the lens galaxy $z=0.89, N_{\mathrm{H} \text {,ion }}$ in the column density of the warm gas at redshift of the source $z=2.51$, both are in $10^{22} \mathrm{~cm}^{-2}$; ${ }^{(2)}$ in $\mathrm{erg} \mathrm{cm} \mathrm{s}^{-1}$; ${ }^{(3)}$ in $10^{-11} \mathrm{erg} \mathrm{cm}^{-2} \mathrm{~s}^{-1}$.
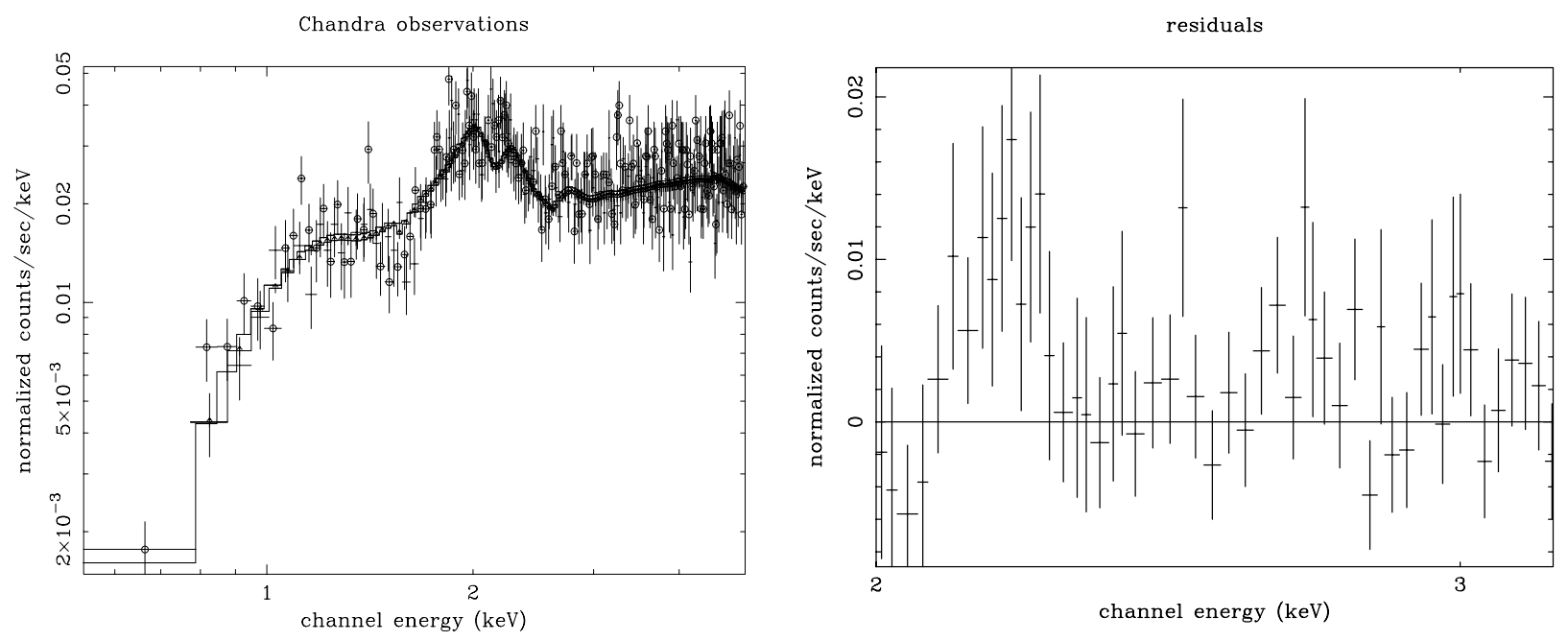

Fig. 4. In the left panel we show the Chandra spectra of both 2000 and 2001 observations with the folded model characterized by a power-law absorbed by a cold gas at the redshift of the lens galaxy. The data taken in 2001 are plotted with a circle. A zoom of the residuals in the line feature region, from Chandra ACIS-S, is shown in the right panel.

optical points have been derived from the photometric data of the NE component given by Courbin et al. (2002), multiplied by 2 to take into account the obscured contribution from the SW component. These data have been corrected for an equivalent galactic absorption $A_{\mathrm{V}}=6.0$, slightly smaller than the $N_{\mathrm{H}}$ value expected from the Chandra data best fit. The galactic contribution to $A_{V}$, estimated from $E(B-V)=0.46$ (Schlegel et al. 1998), is $1.44(R=3.1)$, whereas the additional absorption from the lens galaxy is of the order of 4 mag (Winn et al. 2002). We recall that optical images show that the SW component is much more highly absorbed with a differential reddening of $E(B-V) \simeq 2.75$ (Courbin et al. 2002).

A further problem is the magnification factor of the flux due to the gravitational lens. Following the numerical calculations for the lens modelling by Nair et al. (1993), we assumed the magnification factor for the summed flux of both components to be equal to 10 (see also Mathur \& Nair 1997). A lower factor, of course, would increase the intrinsic luminosity of PKS 1830-211.

We then tried to apply some emission models to reproduce the SED according to the largely accepted scenarios used for high luminosity Blazars. First, we used a single zone homogeneous Synchrotron-Self Compton (SSC) model, but it failed to reproduce both the radio-optical bump and the $\mathrm{X}$ and $\gamma$-ray bump. The SSC model contains a number of free parameters whose values were chosen on the basis of the current blazar physics: we assumed a beaming factor $\delta=16$ and a mean magnetic field $B=0.8 \mathrm{G}$, comparable to those considered for 3C 279 (Hartman et al. 2001). The very high beamed luminosity, of $\sim 4 \times 10^{48} \mathrm{erg} \mathrm{s}^{-1}$, after the correction for the lens magnification, requires a population of emitting electrons confined inside a volume of about $10^{53} \mathrm{~cm}^{3}$ and an electron density of $850 \mathrm{~cm}^{-3}$. The steady electron energy distribution is a broken power law with the break at the Lorentz factor $\gamma_{\mathrm{b}} \simeq 70$, obtained by equating the electron cooling time to the crossing time through the emitting volume. The two spectral indices are $p_{1}=1.80$ for $\gamma<\gamma_{\mathrm{b}}$ and $p_{2}=2.80$ above the break. These values were chosen to match both $\mathrm{X}$ and $\gamma$-ray data, and their difference is that expected from radiative cooling. The maximum Lorentz factor is 2500 , intended to reproduce the decline in the synchrotron spectrum at optical-UV energies.

The resulting SED is plotted in Fig. 6. Note that the synchrotron self absorption frequency (defined by unitary optical depth) is around $3 \times 10^{11} \mathrm{~Hz}$ in the observer's frame and therefore there is no way to account for the emission in the $\mathrm{GHz}$ band. The same problem, found for other blazars like 3C 279 (Hartman et al. 2001) and PKS 1127-145 (Blazejowski et al. 2004), can be solved assuming a multicomponent model in which the radio emission comes from an outer region where the magnetic field is lower. Notice, however, that this SSC model 
PKS 1830-211. Chandra \& INTEGRAL

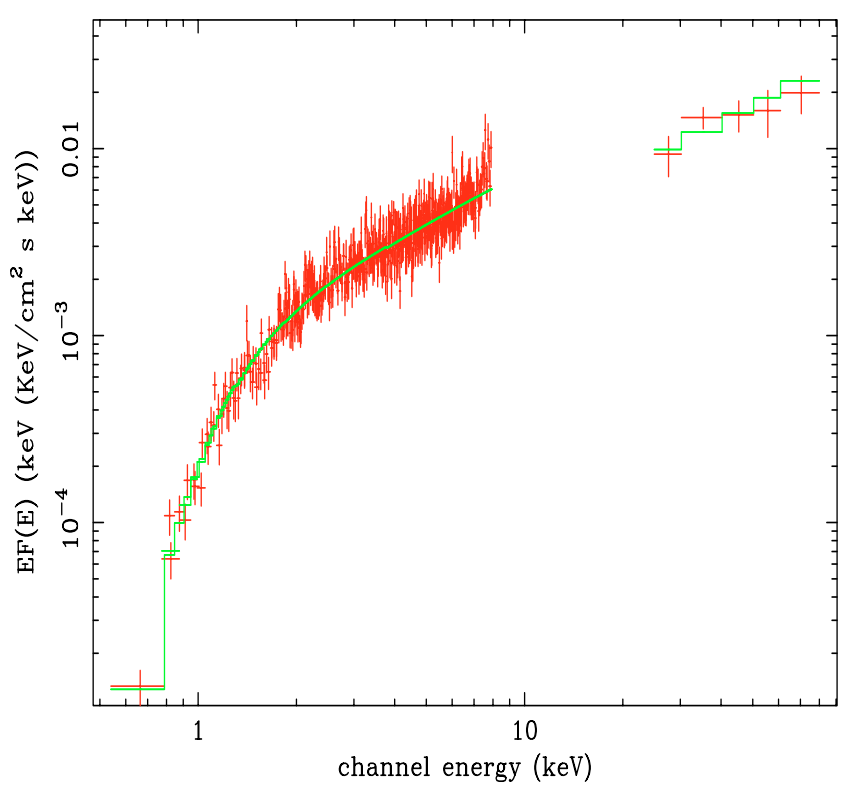

Fig. 5. Broad-band spectra of PKS 1830-211 from Chandra and INTEGRAL. The observations have been performed about one year apart. The data are reproduced with a power-law with $\Gamma \simeq 1.1 \mathrm{ab}-$ sorbed at $z=0.89$ by $N_{\mathrm{H}} \simeq 2 \times 10^{22} \mathrm{~cm}^{-2}$ (in addition to the Galactic absorption at $z=0$ ).

gives hard $\mathrm{X}$ and $\gamma$-ray luminosities lower than the observed values by more than an order of magnitude.

External Radiation Compton (ERC) models consider that the main sources of seed photons are the accretion disk around the massive black hole, the broad line region and, at larger distances, the dust torus. UV photons from the disk are seen deboosted by the relativistic electrons moving outward in the jet from behind and their contribution is small. When applying an ERC model, considering only photons originating in an accretion disk with a luminosity of $4 \times 10^{48} \mathrm{erg} \mathrm{s}^{-1}$ and a multitemperature spectrum $\left(T_{\max }=3.5 \times 10^{5} \mathrm{~K}\right.$, Shakura \& Sunyaev 1973), we obtain a Compton bump much lower than that in the data. Moreover, photons scattered by the BLR give another relevant but insufficient contribution to the IC bump. An increase of $\gamma_{\mathrm{b}}$ would move the peak frequency above $10^{23} \mathrm{~Hz}$ which is in conflict with the steep spectrum observed in the EGRET range.

Perhaps more relevant are photons produced by matter at a distance of a few pc and illuminated by the disk, like the IR emission from a dusty torus at a temperature of $10^{3} \mathrm{~K}$ expected in similar sources (Sikora et al. 2002, see next section). Given the geometry of the jet-disk environment, and the positioning of the emitting volume at a distance of $\sim 10^{19} \mathrm{~cm}$ from the accretion disk, we find that the comoving energy density of the IR external photon field from the dust torus overwhelms the other soft field at least by an order of magnitude. The resulting IC bump is able to reproduce both the $\mathrm{X}$ and $\gamma$-ray continuum. We recall, however, that the latter data are not a strong constraint because of source variability, which in this range is at least a factor of 4 , as indicated from the 3EG catalogue (Hartman et al. 1999).

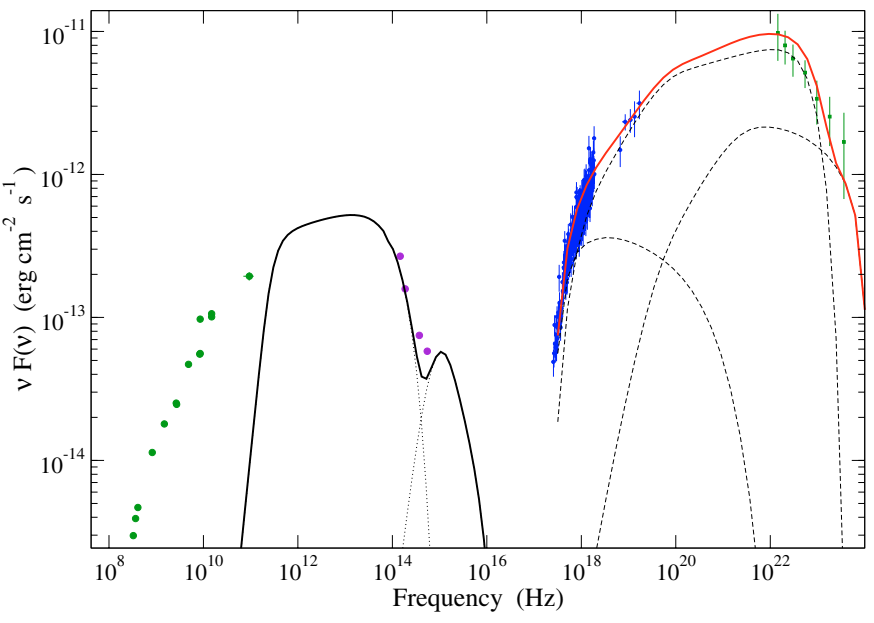

Fig. 6. The Spectral Energy Distribution of PKS 1830-211 in the observers' frame derived from our results and other published data. Radio measurements are from Pramesh Rao \& Subrahmanyan (1988), IR and optical data from Courbin et al. (2002) and $\gamma$-ray data from the EGRET public data archive. Solid lines represent the SED as the sum of various emission contributions computed with the parameters given in the text; dotted lines are the individual low energy contributions: synchrotron emission and accretion disk; dashed lines are high energy spectra of SSC (lower bump) and ERC components.

\section{Discussion}

It is interesting to compare the properties of this distant and lensed blazar with those of other sources of the same class. The SED of Fig. 6 shows that the largest energy output is in the prominent IC bump. A relevant finding of our analysis is that the X-ray spectrum of PKS $1830-211$ is remarkably flat up to energies of $\sim 80 \mathrm{keV}$, while the spectrum above $\sim 100 \mathrm{MeV}$ is much steeper. The difference between these two spectral indices is around 1.5. The peak frequency, however, is not well constrained by the data: in the model described in the previous section it lies around $10^{22} \mathrm{~Hz}$, however it could be lower, in the $\mathrm{MeV}$ range, for a different electron spectrum. PKS 1830-211 can be considered a member of the small subclass of "MeV blazars" discovered by COMPTEL (Bloemen et al. 1995), which are characterised by flat hard X-ray and rather steep $\gamma$-ray spectra (Blom et al. 1996; Tavecchio et al. 2000). Sikora et al. (2002) proposed that the prominent IC bump seen in this type of blazar can be explained by ERC dominated by the interactions of relativistic electrons with the near-IR photons from a hot dusty torus. Our SED modelling of the previous section confirms this scenario also in the case of PKS 1830-211. SSC emission is indeed found to be insufficient to reproduce the IC bump and the ERC emission on optical-UV photons from the BLR gives too many energetic $\gamma$ rays.

A complete understanding of the emission properties of PKS 1830-211, however, presents some difficulties arising from the presence of the gravitational lens. Radio, optical and $\mathrm{X}$-ray data do not give a consistent picture of the absorption of the two lensed components. The large brightness difference observed by Courbin et al. (2002) in HST images was explained by a differential extinction $\Delta A_{\mathrm{V}} \simeq 8.5$. One can expect 
that X-ray images at energies lower than $2 \mathrm{keV}$ would show a SW component much weaker than the NE, however this effect is not observed in our data (see Sect. 2). Spatially resolved high resolution radio spectroscopy of $\mathrm{HCO}$ and $\mathrm{HCN}$ transitions at the lens redshift (Swift et al. 2001) showed that there is no absorption in the NE image, while the SW one presents optically thick absorption with a complex structure. Molecular clouds on the radiation path of the SW component can then be responsible for the differential absorption, however the dust to gas density ratio should be lower than the mean galactic value to account for the much higher optical extinction.

The spectral behaviour of PKS 1830-211 at low X-ray energies is characterized by strong absorption. In Fig. 3 we show the spectrum, in the rest frame, of the combined Chandra observations as the ratio data to model assuming a power-law absorbed by Galactic gas. The ratio is not affected by instrumental response and Galactic absorption. We found that the spectrum below 5-6 keV (source frame) flattens, indicating absorption in excess of the Galactic one, probably due to the lens galaxy at $z=0.89$ and with a column density $N_{\mathrm{H}} \sim 10^{22} \mathrm{~cm}^{-2}$. However a remarkably similar shape is shown in Fig. 5 of Yuan et al. (2003) in the case of the XMM-Newton observations of RX J1028.6-0844 ( $z=4.276)$, GB1428+4217 $(z=4.72)$ and PMN J0525-3343 $(z=4.4)$. The energy of the break is similar in all sources, with a power-law photon index $\Gamma \simeq 1$. This evidence strongly supports a common scenario in all these high redshift sources where absorption plays a role. The most plausible hypothesis (Fabian et al. 2001), includes the presence of a warm gas in the nuclear region (similar to that observed in Sy 1 galaxies). This warm gas is intrinsic to the source and is characterized by a column density $N_{\mathrm{H}, z} \sim 10^{23} \mathrm{~cm}^{-2}$. In the case of PKS 1830-211 this model seems to be supported by marginal evidence of an emission line at the observed energy $2.15 \mathrm{keV}$, that can be interpreted as a blueshifted iron line $(v / c \simeq 0.18)$.

Further Chandra and XMM-Newton observations will help to understand the nature of the absorption in PKS 1830-211 and in the other radio-loud quasars at high redshift.

Acknowledgements. A.D.R. would like to thank A. Bazzano and P. Ubertini for useful discussions. We acknowledge financial support by ASI (Italian Space Agency) via contract I/R/041/02. Part of this work was performed with the financial support Universitá La Sapienza di Roma.

\section{References}

Bassani, L., Malizia, A., Stephen, J. B., et al. 2004, Proc. V INTEGRAL Workshop, Munich February 2004, ESA SP-552 [arXiv: astro-ph/040442]

Bird, A. J., Barlow, E. J., Bassani, L., et al. 2004, ApJ, 607, L33

Blazejowski, M., Siemiginowska, A., Sikora, M., Moderski, R., \& Bechtold, J. 2004, ApJ, 600, L27

Bloemen, H., Bennet, K., Blom, J. J., et al. 1995, A\&A, 293, L1

Blom, J. J., Bennet, K., Bloemen, H., et al. 1996, A\&AS, 120, 507

Celotti, A., \& Fabian, A. C. 1993, MNRAS, 264, 128

Courbin, F., Meylan, G., Kneib, J. P., \& Lidman, C. 2002, ApJ, 575, 95

Fabian, A. C., Celotti, A., Iwasawa, K., et al. 2001, MNRAS, 323, 373

Fiore, F., Elvis, M., Giommi, P., \& Padovani, P. 1998, ApJ, 492, 79

Goldwurm, A., David, P., Foschini, L., et al. 2003, A\&A, 411, L223

Hartman, R. C., Bertsch, D. L., Bloom, S. D., et al. 1999, ApJS, 123, 79

Hartman, R. C., Böttcher, M., Aldering, G., et al. 2001, ApJ, 553, 683

Lidman, C., Courbin, F., Meylan, G., et al. 1999, ApJ, 514, L57

Lovell, J. E., Jauncey, D., Reynolds, J. E., et al. 1998, ApJ, 508, L51

Mathur, S., \& Nair, S. 1997, ApJ, 484, 140

Mattox, J. R., Schachter, J., Molnar, L., Hartman, R. C., \& Patnaik, A. R. 1997, ApJ, 481, 95

Nair, S., Narashima, D., \& Pramesh Rao, A. 1993, ApJ, 407, 46

Oshima, T., Mitsuda, K., Ota, N., et al. 2001, ApJ, 551, 929

Pramesh Rao, A., \& Subrahmanyan, R. 1988, MNRAS, 231, 229

Revnivtsev, M. G., Sunyaev, R. A., Varshalovich, D. A., et al. 2004, Astron. Lett., 30, 382

Reeves, J. N., \& Turner, M. J. L. 2000, MNRAS, 316, 234

Shakura, N. I., \& Sunyaev, R. A. 1973, A\&A, 24, 337

Schlegel, D. J., Finkbeiner, D. P., \& Davis, M. 1998, ApJ, 500, 525

Sikora, M., Madjeski, G., Moderski, R., \& Poutanen, J. 1997, ApJ, 484, 108

Sikora, M., \& Blazejowski, M. 2002, ApJ, 577, 78

Stark, A. A., Gammie, C. F., Wilson, R. W., et al. 1992, ApJS, 79, 77

Swift, J. J., Welch, W. J., \& Frye, B. L. 2001, ApJ, 548, L29

Terrier, R., Lebrun, F., \& Bazzano, A. 2003, A\&A, 411, L167

Tavecchio, F., Maraschi, L., Ghisellini, G., et al. 2000, ApJ, 543, 535

Wiklind, T., \& Combes, F. 1996, Nature, 379, 139

Winn, J. M., Kochanek, C. S., McLeod, B. A., et al. 2002, ApJ, 575, 103

Worsley, M. A., Fabian, A. C., Turner, A. K., Celotti, A., \& Iwasawa, K. 2004, MNRAS, 350, 207

Yuan, W., Fabian, A. C., Celotti, A., \& Jonker, P. G. 2003, MNRAS, 346, L7 